\title{
Great enhancement of Curie temperature and magnetic anisotropy in two-dimensional van der Waals magnetic semiconductor heterostructures
}

\author{
Xue-Juan Dong ${ }^{1}$, Jing-Yang You ${ }^{1}$, Zhen Zhang ${ }^{1}$, Bo $\mathrm{Gu}^{2,3},{ }^{*}$ and Gang $\mathrm{Su}^{2,3,1 \dagger}$ \\ ${ }^{1}$ School of Physical Sciences, University of Chinese Academy of Sciences, Beijing 100049, China \\ ${ }^{2}$ Kavli Institute for Theoretical Sciences, and CAS Center for Excellence in Topological Quantum Computation, \\ University of Chinese Academy of Sciences, Beijing 100190, China \\ ${ }^{3}$ Physical Science Laboratory, Huairou National Comprehensive Science Center, Beijing 101400, China
}

(Dated: September 2, 2020)

\begin{abstract}
In two-dimensional (2D) magnetic systems, large magnetic anisotropy is needed to stabilize the magnetic order according to Mermin-Wagner theorem. Based on density functional theory (DFT) calculations, we propose that the magnetic anisotropic energy (MAE) of $2 \mathrm{D}$ ferromagnetic (FM) semiconductors can be strongly enhanced in van der Waals heterostructures by attaching a nonmagnetic semiconductor monolayer with large spin-orbit coupling. We studied $\mathrm{Cr}_{2} \mathrm{Ge}_{2} \mathrm{Te}_{6} / \mathrm{PtSe}_{2}$ bilayer heterostructures, where each layer has been realized in recent experiments. The DFT calculations show that the MAE of $\mathrm{Cr}_{2} \mathrm{Ge}_{2} \mathrm{Te}_{6} / \mathrm{PtSe}_{2}$ is enhanced by $70 \%$, and the Curie temperature $T_{C}$ is increased far beyond room temperature. A model Hamiltonian is suggested to analyze the DFT results, showing that both the Dzyaloshinskii-Moriya interaction and the single-ion anisotropy contribute to the enhancement of the MAE. Based on the superexchange picture, we find that the decreased energy difference between $3 \mathrm{~d}$ orbitals of $\mathrm{Cr}$ and $5 \mathrm{p}$ orbitals of Te contributes partially to the increase of $T_{C}$. Our present work indicates a promising way to enhance the MAE and $T_{C}$ by constructing van der Waals semiconductor heterostructures, which will inspire further studies on the $2 \mathrm{D}$ magnetic semiconductor systems.
\end{abstract}

\section{INTRODUCTION}

To achieve room temperature ferromagnetic semiconductors has long been an important topic in science [1]. Recently, magnetism in two-dimensional (2D) van der Waals materials has become a hot-spot which could lead to new device applications in information storage and spintronics [2-4]. People have devoted to explore 2D van der Waals ferromagnetic semiconductors, such as $\mathrm{Cr}_{2} \mathrm{Ge}_{2} \mathrm{Te}_{6}$ [5], $\mathrm{CrI}_{3}$ [6], $\mathrm{Fe}_{3} \mathrm{GeTe}_{2}$ [7], $\mathrm{VSe}_{2}$ [8], $\mathrm{MnSe}_{2}$ $[9]$, and $\mathrm{FePS}_{3}[10,11]$, etc. These layered materials have enriched the database of $2 \mathrm{D}$ ferromagnetic materials, but there are still challenges ahead. On one hand, large magnetic anisotropic energy (MAE) is required to suppress the thermal fluctuations and to stabilize the 2D magnetic moment according to Mermin-Wagner theorem [12]. Different pictures, for instance, Kitaev interaction, single-ion anisotropy (SIA) [13] and p-d covalency [13] have been suggested to understand the MAE in 2D van der Waals ferromagnetic semiconductors. The Kitaev interaction and the SIA are originated from the spin-orbit coupling (SOC), the latter as a relativistic effect is usually small in many materials. On the other hand, high Curie temperature $T_{C}$ above room temperature is very required from practical applications [14]. The Curie temperature of $\mathrm{Cr}_{2} \mathrm{Ge}_{2} \mathrm{Te}_{6}$ bilayer and $\mathrm{CrI}_{3}$ monolayer is 28 $\mathrm{K}$ and $45 \mathrm{~K}$, respectively, far below room temperature $[5,6]$. Efforts have been paid to enhance the magnetization of $2 \mathrm{D}$ van der Waals magnets and to seek for new 2D magnetic material as well as to control the magnetism

\footnotetext{
* gubo@ucas.ac.cn

† gsu@ucas.ac.cn
}

using mechanic and electronic means $[15,16]$. Strain is another useful way to control the magnetism in $2 \mathrm{D}$ ferromagnetic semiconductors. For example, $T_{C}$ in $\mathrm{Cr}_{2} \mathrm{Ge}_{2} \mathrm{Se}_{6}$ monolayer can be increased beyond room temperature by applying just a few percent tensile strain [17], and the MAE of $\mathrm{CrI}_{3}$ monolayer can also be tuned by strain [18].

It is known that combining two kinds of $2 \mathrm{D}$ materials into heterostructures could regulate the overall performance [19-22], which can thus provide platforms to study interfaces and device applications [23-25]. Van der Waals heterostructures are found to be an exotic platform that could realize fascinating phenomena [26-29]. The emergence of 2D magnetic materials has facilitated to explore more functional heterostructures, e.g. multiferroicity [30] and tunable electronic structure [31].

In $\mathrm{WSe}_{2} / \mathrm{CrI}_{3}$ heterostructures, the degenerate valleys in $\mathrm{WSe}_{2}$ are split due to the proximity effect, where a large magnetic field of $13 \mathrm{~T}$ can be obtained by means of the ferromagnetic layer $\mathrm{CrI}_{3}[32]$. The chiral edge state is manipulated by constructing $\mathrm{WTe}_{2} /$ bilayer $\mathrm{CrI}_{3} / \mathrm{WTe}_{2}$ heterostructures, in which $\mathrm{CrI}_{3}$ was used to magnetize the topological insulator $\mathrm{WTe}_{2}$ [33]. In $\mathrm{FeI}_{2} / \mathrm{In}_{2} \mathrm{Se}_{3}$ heterostructures, it is found that $\mathrm{FeI}_{2}$ could undergo phase transition from ferromagnetic to antiferromagnetic (AFM) phase when the direction of ferroelectric polarization of $\mathrm{In}_{2} \mathrm{Se}_{3}$ is reversed [34]. Strong magnetic proximity effect via the s-d exchange coupling was proposed at the InAs/(Ga,Fe)Sb interface [35]. The effect of magnetic field on magnetic properties of metallic heterostructures $\mathrm{Fe}_{3} \mathrm{O}_{4} / \mathrm{Pt}$ was also studied [36]. The lattice mismatch is important for constructing heterostructures in experiments, and the epitaxial methods were often applied to produce van der Waals for highly lattice-mismatched systems [37]. The heterostructures with large lattice mis- 


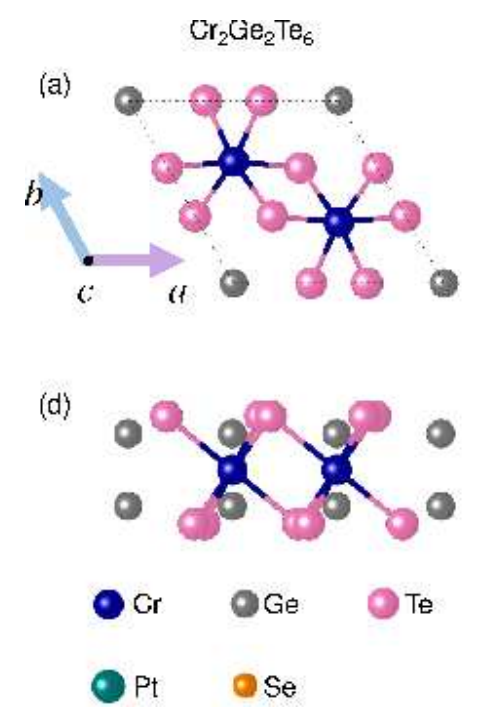

(b)

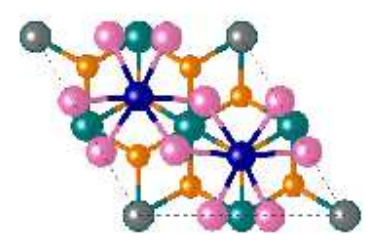

(e)
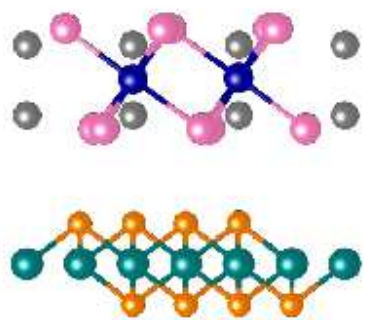

Stack-2

(c)

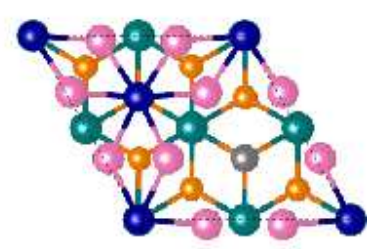

(†)
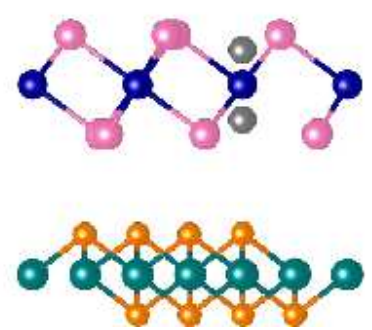

FIG. 1. Crystal structures of two-dimensional $\mathrm{Cr}_{2} \mathrm{Ge}_{2} \mathrm{Te}_{6}$ and $\mathrm{Cr}_{2} \mathrm{Ge}_{2} \mathrm{Te}_{6} / \mathrm{PtSe}_{2}$ heterostructures with Stack-1 and Stack-2, (a)-(c): top view; (d)-(f) side view, respectively.

match about $10 \%$ can be realized in the experiments. For example, the heterostructures GaSe/ $\mathrm{MoSe}_{2}$ with lattice mismatch of $13 \%$ [38] and $\mathrm{FeSe} / \mathrm{Bi}_{2} \mathrm{Te}_{3}$ with lattice mismatch of $19 \%$ [39] were realized in recent experiments.

By constructing 2D van der Waals semiconductor heterostructures, is it possible to enhance the MAE and $T_{C}$ of ferromagnetic semiconductors? To answer this question, in this work, in terms of first principles calculations, we propose an efficient way to enhance the MAE and $T_{C}$ of $\mathrm{Cr}_{2} \mathrm{Ge}_{2} \mathrm{Te}_{6}$, through constructing van der Waals heterostructures with nonmagnetic $\mathrm{PtSe}_{2}$. Both $\mathrm{Cr}_{2} \mathrm{Ge}_{2} \mathrm{Te}_{6}$ and $\mathrm{PtSe}_{2}$ are $2 \mathrm{D}$ van der Waals semiconductors that have already been fabricated in experiments [5, 40]. We consider two stack configurations and find the Curie temperatures $T_{C}$ of two stacks are larger than $600 \mathrm{~K}$, while the MAE is increased by $70 \%$. We present a model and show that both the Dzyaloshinskii-Moriya interaction and the SIA contribute to the enhancement of the magnetic anisotropy. The increase of $T_{C}$ is partially owing to the decreased energy difference between $3 \mathrm{~d}$ orbitals of $\mathrm{Cr}$ and $5 \mathrm{p}$ orbitals of Te. Our work will inspire further studies to enhance the magnetic properties of low-dimensional magnetic materials with the help of 2D van der Waals heterostructures. Considering the great progress of $2 \mathrm{D}$ van der Waals stacking structures in recent experiments, such as the van der Waals stackingdependent interlayer [41], and general synthesis of 2D van der Waals heterostructure arrays [42], our present proposal will be readily feasible for experiments.

\section{CALCULATION METHODS}

We perform first principles calculations using Vienna $a b$ initio simulation package (VASP) [43]. Spinpolarized calculations are conducted with the Perdew-
Burke-Ernzerhof exchange-correlation function and the generalized-gradient approximation. We adopted the DFT-D2 method of Grimme to describe the van der Waals interaction between $\mathrm{Cr}_{2} \mathrm{Ge}_{2} \mathrm{Te}_{6}$ and $\mathrm{PtSe}_{2}$ layers [44]. Spin-orbit coupling is taken into account in the calculations. $5 \times 5 \times 1$ and $3 \times 3 \times 1$ Mohnkhorst-Pack $k$-mesh sampling grids are used for the unit cell and supercell, respectively. The vacuum length is taken as $20 \AA$, which is enough to isolate the present $2 \mathrm{D}$ system. The in-plane supercell is taken as $\sqrt{3} \times 1$ in a rectangular shape. The Hubbard $U$ for $3 \mathrm{~d}$ electrons of $\mathrm{Cr}$ is chosen as $4 \mathrm{eV}$, which should be reasonable [17]. We relaxed the structure of $\mathrm{Cr}_{2} \mathrm{Ge}_{2} \mathrm{Te}_{6}$ and $\mathrm{PtSe}_{2}$ to obtain the groundstate structure. Based on the DFT results, the Curie temperature is calculated by using the Monte Carlo simulations [45] based on the 2D Ising model, where a $60 \times$ 60 supercell is adopted, and $10^{5}$ steps are performed for every temperature to acquire the equilibrium.

\section{III. $\mathrm{CR}_{2} \mathrm{GE}_{2} \mathrm{TE}_{6} / \mathrm{PTSE}_{2}$ HETEROSTRUCTURES AND ENHANCEMENT OF MAGNETISM}

Bulk $\mathrm{Cr}_{2} \mathrm{Ge}_{2} \mathrm{Te}_{6}$ has been synthesized in 1995 [46] and successfully exfoliated into layers recently [5]. The spacegroup of $2 \mathrm{D} \mathrm{Cr}_{2} \mathrm{Ge}_{2} \mathrm{Te}_{6}$ is $\mathrm{P} \overline{3} 1 \mathrm{~m}$ (No.162). Monolayer $\mathrm{PtSe}_{2}$ is considered as the most appropriate candidate to combine with $2 \mathrm{D} \mathrm{Cr}_{2} \mathrm{Ge}_{2} \mathrm{Te}_{6}$, which is a semiconductor with a lattice constant of $3.76 \AA$ that is well matched with the lattice of $\mathrm{Cr}_{2} \mathrm{Ge}_{2} \mathrm{Te}_{6}$ and can be obtained by mechanical exfoliation methods [40]. The space group of $\mathrm{PtSe}_{2}$ is $\mathrm{P} \overline{3} \mathrm{~m} 1$ (No. 164), very similar to that of $\mathrm{Cr}_{2} \mathrm{Ge}_{2} \mathrm{Te}_{6}$. The heterostructure is constructed in such a way that monolayer $\mathrm{PtSe}_{2}$ with a size of $2 \times 2$ is attached to monolayer $\mathrm{Cr}_{2} \mathrm{Ge}_{2} \mathrm{Te}_{6}$ with a size of $1 \times 1$. The lattice mismatch between $\mathrm{Cr}_{2} \mathrm{Ge}_{2} \mathrm{Te}_{6}$ and $\mathrm{PtSe}_{2}$ is $10 \%$, 
TABLE I. The DFT results of lattice parameter and electronic and magnetic properties of considered $\mathrm{Cr}_{2} \mathrm{Ge}_{2} \mathrm{Te}_{6}$ with various lattice constants, and $\mathrm{Cr}_{2} \mathrm{Ge}_{2} \mathrm{Te}_{6} / \mathrm{PtSe}_{2}$ heterostructures with Stack-1 and Stack-2 obtained by the GGA $+\mathrm{SOC}+U$ calculations, where $\mathrm{U}=4 \mathrm{eV}$.

\begin{tabular}{lc|ccc|cc}
\hline \hline \multicolumn{5}{c|}{} & \multicolumn{3}{c|}{$\mathrm{Cr}_{2} \mathrm{Ge}_{2} \mathrm{Te}_{6}$} & \multicolumn{2}{c}{$\mathrm{Cr}_{2} \mathrm{Ge}_{2} \mathrm{Te}_{6} / \mathrm{PtSe}_{2}$} \\
\hline & Expt. & Expt. lat. & Relax lat. & Strain lat. & Stack-1 & Stack-2 \\
\hline lattice $(\AA)$ & 6.83 & 6.83 & 6.96 & 7.22 & 7.22 & 7.22 \\
band gap $(\mathrm{eV})$ & - & 0.03 & 0.14 & 0.36 & 0.39 & 0.45 \\
$E_{F M z}(\mathrm{eV})$ & - & -44.5662 & -44.6458 & -44.4594 & -109.3624 & -109.2307 \\
$E_{F M x}(\mathrm{eV})$ & - & -44.5650 & -44.6445 & -44.4575 & -109.3601 & -109.2284 \\
$E_{A F M}(\mathrm{eV})$ & - & -44.4953 & -44.5399 & -44.3027 & -109.1711 & -109.0484 \\
$\mathrm{~S}_{d}\left(\mu_{B}\right)(\mathrm{Cr})$ & - & 3.59 & 3.52 & 3.66 & 3.59 & 3.68 \\
$\mathrm{MAE}(\mathrm{meV})$ & - & 1.19 & 1.33 & 1.89 & 2.32 & 2.23 \\
$T_{C}(\mathrm{~K})$ & 28 & 30 & 200 & 464 & 693 & 625 \\
\hline \hline
\end{tabular}

which could be executable in experiment for $2 \mathrm{D}$ van der Waals materials heterostructures [19]. We propose two ways to make the heterostructures named as Stack-1 and Stack-2, in both configurations $\mathrm{PtSe}_{2}$ is located under $\mathrm{Cr}_{2} \mathrm{Ge}_{2} \mathrm{Te}_{6}$. In Stack-1, the center of $\mathrm{Cr}_{2} \mathrm{Ge}_{2} \mathrm{Te}_{6}$ unit cell and the $2 \times 2 \mathrm{PtSe}_{2}$ is aligned in $c$ direction, while Stack-2 can be treated as Stack-1 translating $\mathrm{Cr}_{2} \mathrm{Ge}_{2} \mathrm{Te}_{6}$ layer with $1 / 3$ unit cell along $\left[\begin{array}{lll}1 & 2 & 0\end{array}\right]$ direction. The spacegroup for Stack-1 and Stack-2 structures is P3 (No.143). The crystal structure of monolayer $\mathrm{Cr}_{2} \mathrm{Ge}_{2} \mathrm{Te}_{6}$ and the two stacks are shown in Fig. 1. It is noted that the lattice constant becomes $7.22 \AA$ for the heterostructure $\mathrm{Cr}_{2} \mathrm{Ge}_{2} \mathrm{Te}_{6} / \mathrm{PtSe}_{2}$, which is equivalent to applying $3.7 \%$ tensile strain to monolayer $\mathrm{Cr}_{2} \mathrm{Ge}_{2} \mathrm{Te}_{6}$.

The properties of $\mathrm{Cr}_{2} \mathrm{Ge}_{2} \mathrm{Te}_{6}$ are sensitive to its lattice constant [30]. For $2 \mathrm{D} \mathrm{Cr}_{2} \mathrm{Ge}_{2} \mathrm{Te}_{6}$, the lattice constant is $6.83 \AA$ in the experiment [5], and becomes $6.96 \AA$ in the optimized structure. The experimental and the calculated results with experimental and relaxed lattice constants are given in Table I. The experimental data of $\mathrm{T}_{C}$ and lattice constant for $2 \mathrm{D}_{2} \mathrm{Ge}_{2} \mathrm{Te}_{6}$ shown in Table I are taken from Ref. [5]. For the hexagonal lattice, we used the following three configurations for AFM ordering: the AFM Néel, AFM stripe, and AFM zigzag states [17]. The AFM state used for discussions in Table I is the AFM zigzag state, which is the lowest energy AFM state according to our calculations. In addition, for the bulk $\mathrm{Cr}_{2} \mathrm{Ge}_{2} \mathrm{Te}_{6}$, there are some experimental reports, for example, the MAE is $0.25 \mathrm{meV}$ per unit cell of $\mathrm{Cr}_{6} \mathrm{Ge}_{6} \mathrm{Te}_{18}$ [47], $\mathrm{T}_{C}$ is about $66 \mathrm{~K}$ [47] and $62.5 \mathrm{~K}$ [48], and the spin moment is $3.41 \mu_{B}$ [48].

The MAE is defined by the energy difference of the inplane and the out-of-plane ferromagnetic state and can be expressed as $M A E=E_{F M x}-E_{F M z}$, where $E_{F M x}$ and $E_{F M z}$ denote the energy of the ferromagnetic state with in-plane and out-of-plane direction of the unit cell, respectively. Our results suggest that these materials possess a ferromagnetic ground state with out-of-plane magnetization.

Our calculations show that $\mathrm{Cr}_{2} \mathrm{Ge}_{2} \mathrm{Te}_{6} / \mathrm{PtSe}_{2}$ heterostructure Stack-1 and Stack-2 are semiconductors with finite band gaps of 0.39 and $0.45 \mathrm{eV}$, respectively. The electronic band structures and atom-projected partial density of states of Stack-1 and Stack-2 are presented in Fig. 2.

The magnetization as a function of temperature obtained by Monte Carlo simulations based on 2D Ising model is depicted in Fig. 3(a). One may note that our calculated result for $T_{C}$ of monolayer $\mathrm{Cr}_{2} \mathrm{Ge}_{2} \mathrm{Te}_{6}$ agrees well with the experimental result, revealing that our calculating method is reliable. It is shown that the Curie temperature $T_{C}$ of $\mathrm{Cr}_{2} \mathrm{Ge}_{2} \mathrm{Te}_{6}$ with $3.7 \%$ tensile strain (the lattice constant $7.22 \AA$ ) is increased beyond room temperature, and $T_{C}$ of the heterostructures with Stack1 and Stack- 2 is increased above $600 \mathrm{~K}$. In addition to the increase of Curie temperature, the MAE is also increased by about $40 \%$ in the $\mathrm{Cr}_{2} \mathrm{Ge}_{2} \mathrm{Te}_{6}$ with $3.7 \%$ tensile strain, and enhanced by about $70 \%$ in the heterostructures with Stack-1 and Stack-2 as shown in Fig. 3(b). It is indicated that the MAE of Stack-1 and Stack-2 are both larger than that of $\mathrm{Cr}_{2} \mathrm{Ge}_{2} \mathrm{Te}_{6}$ monolayer, showing that the nonmagnetic layer $\mathrm{PtSe}_{2}$ has a remarkable effect on the enhancement of magnetism in $2 \mathrm{D} \mathrm{Cr}_{2} \mathrm{Ge}_{2} \mathrm{Te}_{6}$, which will be discussed in next section.

To study the effect of Hubbard parameter $\mathrm{U}$ on the calculated $T_{C}$ and MAE, we perform DFT calculations for different values of $U$, as shown in Fig. 4. One may see that with increasing $U, T_{C}$ of Stack-1 and Stack-2 remain almost unaltered, while $T_{C}$ of monolayer $\mathrm{Cr}_{2} \mathrm{Ge}_{2} \mathrm{Te}_{6}$ is decreasing with the increase of $U$. However, with increasing $\mathrm{U}$ the MAE is found to increase for monolayer $\mathrm{Cr}_{2} \mathrm{Ge}_{2} \mathrm{Te}_{6}$ and heterostructure Stack-1 and Stack-2, revealing that the enhancements of $T_{C}$ and MAE have different mechanisms, and electron interactions play important roles in the enhancement of MAE. In addition, the U-dependent band gap is also studied by DFT calculations. Our results show that the band gap slightly increases with increasing $\mathrm{U}$ in the range of $4-5 \mathrm{eV}$, as this is typical for $3 \mathrm{~d}$ electrons. 

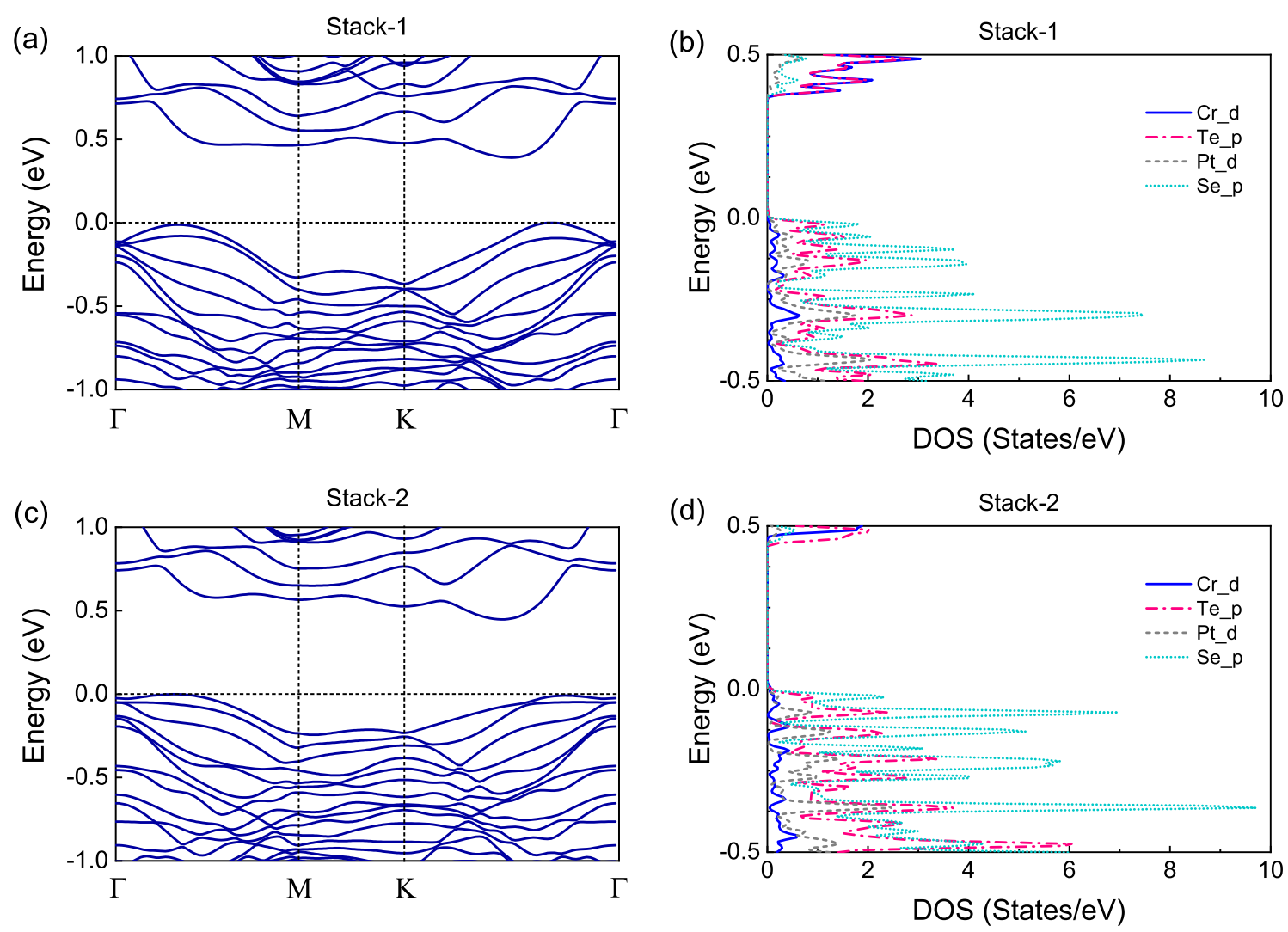

FIG. 2. Electronic band structures and partial DOS of two-dimensional $\mathrm{Cr}_{2} \mathrm{Ge}_{2} \mathrm{Te}_{6} / \mathrm{PtSe} \mathrm{e}_{2}$ heterostructures with (a), (b) Stack-1 and (c), (d) Stack-2, respectively, obtained by the GGA + SOC $+U$ calculations with $\mathrm{U}=4 \mathrm{eV}$.

\section{THEORETICAL ANALYSIS}

To describe the magnetic anisotropy in monolayer $\mathrm{Cr}_{2} \mathrm{Ge}_{2} \mathrm{Te}_{6}$ and the heterostructure $\mathrm{Cr}_{2} \mathrm{Ge}_{2} \mathrm{Te}_{6} / \mathrm{PtSe}_{2}$, we write down a general spin Hamiltonian
TABLE II. The diagonal elements of exchange coupling matrix $\mathrm{J}$ (in units of meV) for monolayer $\mathrm{Cr}_{2} \mathrm{Ge}_{2} \mathrm{Te}_{6}, 2 \mathrm{D}$ $\mathrm{Cr}_{2} \mathrm{Ge}_{2} \mathrm{Te}_{6}$ with $3.7 \%$ tensile strain and $\mathrm{Cr}_{2} \mathrm{Ge}_{2} \mathrm{Te}_{6} / \mathrm{PtSe}$ heterostructures calculated by the GGA $+\mathrm{SOC}+U$ and the four-state method [53]. Here $\mathrm{U}=4 \mathrm{eV}$.

$$
\begin{aligned}
\hat{H}_{\text {spin }}= & \sum_{<i, j>} J_{x x} S_{i x} S_{j x}+\sum_{<i, j>} J_{x y} S_{i x} S_{j y}+\sum_{<i, j>} J_{x z} S_{i x} S_{j z} \\
& +\sum_{<i, j>} J_{y x} S_{i y} S_{j x}+\sum_{<i, j>} J_{y y} S_{i y} S_{j y}+\sum_{<i, j>} J_{y z} S_{i y} S_{j z} \\
& +\sum_{<i, j>} J_{z x} S_{i z} S_{j x}+\sum_{<i, j>} J_{z y} S_{i z} S_{j y}+\sum_{<i, j>} J_{z z} S_{i z} S_{j z} \\
& +\sum_{i, \alpha, \beta} A_{\alpha \beta} S_{i \alpha} S_{i \beta} .
\end{aligned}
$$

In Eq. (1), $i$ and $j$ denote the magnetic atom sites, and the summation on $\langle i, j\rangle$ only includes the nearest neighbors. $\alpha$ and $\beta$ denote three directions of $x y$ and $z$ axis. The former nine terms in Eq. (1) are the nearest neighbor exchange interactions between different spin components and the last term represents SIA energy. $\quad J_{\alpha \beta}$ are the matrix elements of exchange couplings between the nearest spins, and the off-diagonal

\begin{tabular}{lccc}
\hline \hline & $\mathrm{J}_{x x}$ & $\mathrm{~J}_{y y}$ & $\mathrm{~J}_{z z}$ \\
\hline $\mathrm{Cr}_{2} \mathrm{Ge}_{2} \mathrm{Te}_{6}$-relax & -8.068 & -7.467 & -8.316 \\
$\mathrm{Cr}_{2} \mathrm{Ge}_{2} \mathrm{Te}_{6}$-strain & -11.314 & -10.591 & -11.620 \\
Stack-1 & -12.557 & -11.813 & -12.896 \\
Stack-2 & -12.290 & -11.508 & -12.623 \\
\hline \hline
\end{tabular}

elements lead to the Kitaev interactions $[13,49]$ and the Dzyaloshinskii-Moriya (DM) [50-52] interactions. Matrix elements of exchange coupling matrix $\mathrm{J}$ of $\mathrm{Cr}_{2} \mathrm{Ge}_{2} \mathrm{Te}_{6}$ and $\mathrm{Cr}_{2} \mathrm{Ge}_{2} \mathrm{Te}_{6} / \mathrm{PtSe}_{2}$ could be calculated by the GGA + $\mathrm{SOC}+U$ method and the four-state method [53], where we take, as usual, $\mathrm{U}=4 \mathrm{eV}$.

To calculate parameter $\mathrm{J}_{\alpha \beta}$, for instance, $\mathrm{J}_{x y}$, the following four classical spin configurations are used [53]: $S_{1}$ $=(1,0,0), S_{2}=(0,1,0) ; S_{1}=(1,0,0), S_{2}=(0,-1,0)$; $S_{1}=(-1,0,0), S_{2}=(0,1,0) ; S_{1}=(-1,0,0), S_{2}=(0$, $-1,0)$. Keep the same for the rest spins, where $S=(0$, $0,1)$ is used in our calculation. Eq. (1) with these four 


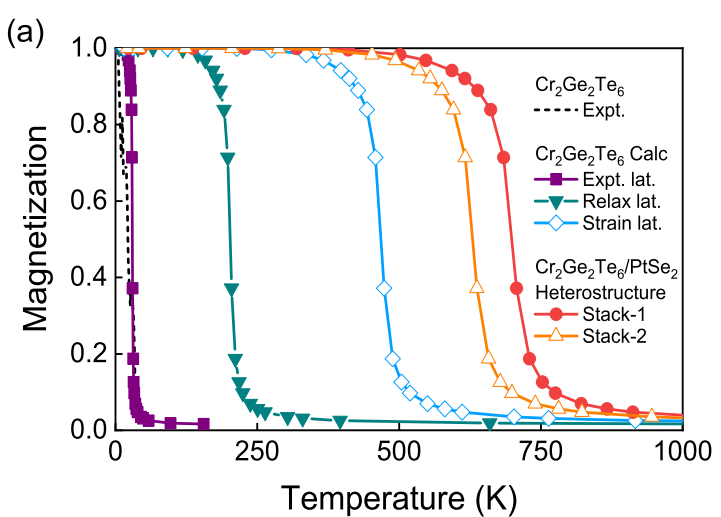

(b)

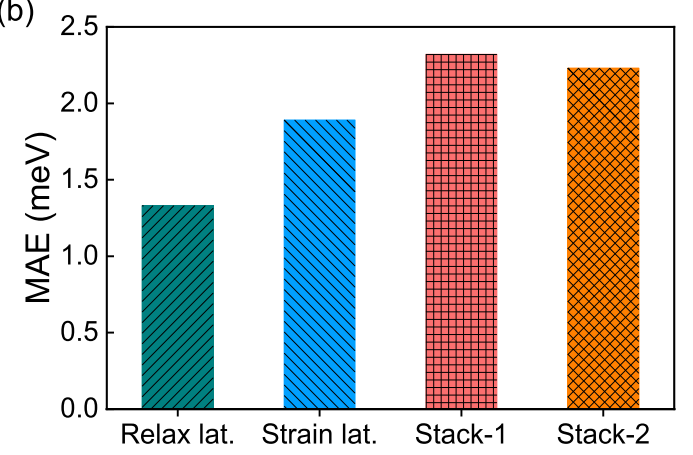

FIG. 3. For two-dimensional $\mathrm{Cr}_{2} \mathrm{Ge}_{2} \mathrm{Te}_{6}, \mathrm{Cr}_{2} \mathrm{Ge}_{2} \mathrm{Te}_{6}$ with strain, and $\mathrm{Cr}_{2} \mathrm{Ge}_{2} \mathrm{Te}_{6} / \mathrm{PtSe}_{2}$ heterostructures with Stack-1 and Stack-2, (a) the normalized magnetization as a function of temperature, in which $\mathrm{Cr}_{2} \mathrm{Ge}_{2} \mathrm{Te}_{6}$ with experimental lattice was taken from our previous work [17] and (b) the magnetic anisotropic energy (MAE). The experimental result of $\mathrm{Cr}_{2} \mathrm{Ge}_{2} \mathrm{Te}_{6}$ is taken from Ref. [5]. The calculated results are obtained by the GGA $+\mathrm{SOC}+U$ calculations and the Monte Carlo simulations, where $\mathrm{U}=4 \mathrm{eV}$.

spin configurations can be written as

$$
\begin{aligned}
& E_{1}=J_{x y} S_{1 x} S_{2 y}+E_{0}=J_{x y}|S|^{2}+E_{0}, \\
& E_{2}=-J_{x y} S_{1 x} S_{2 y}+E_{0}=-J_{x y}|S|^{2}+E_{0}, \\
& E_{3}=-J_{x y} S_{1 x} S_{2 y}+E_{0}=-J_{x y}|S|^{2}+E_{0}, \\
& E_{4}=J_{x y} S_{1 x} S_{2 y}+E_{0}=J_{x y}|S|^{2}+E_{0} .
\end{aligned}
$$

One has $J_{x y}=\left(\left(E_{1}+E_{4}\right)-\left(E_{2}+E_{3}\right)\right) / 4$. Other elements of $\mathrm{J}$ can be obtained in a similar way. The diagonal elements of J are given in Table II. The negative values mean the ferromagnetic coupling. It is clear that the ground state is ferromagnetic for these four structures. The offdiagonal elements of $\mathrm{J}$ and their differences of monolayer $\mathrm{Cr}_{2} \mathrm{Ge}_{2} \mathrm{Te}_{6}$ and $\mathrm{Cr}_{2} \mathrm{Ge}_{2} \mathrm{Te}_{6} / \mathrm{PtSe}_{2}$ heterostructures are given in Table III.

The magnitude of DM interaction can be estimated as

$$
D_{z}=\frac{\left|J_{y x}-J_{x y}\right|}{2} .
$$

(a)

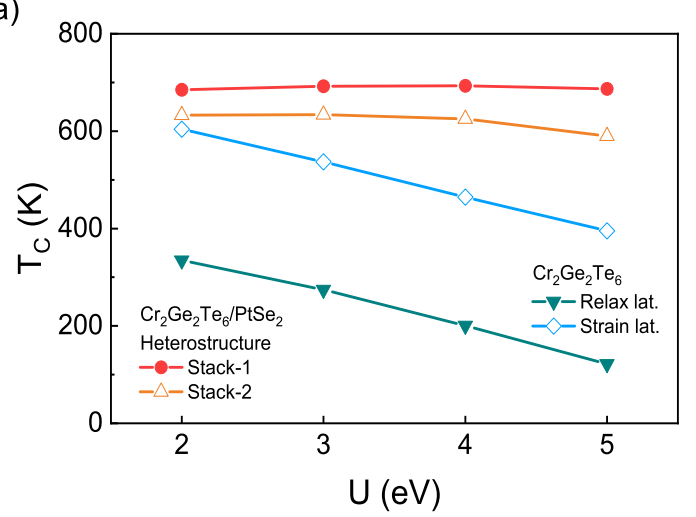

(b)

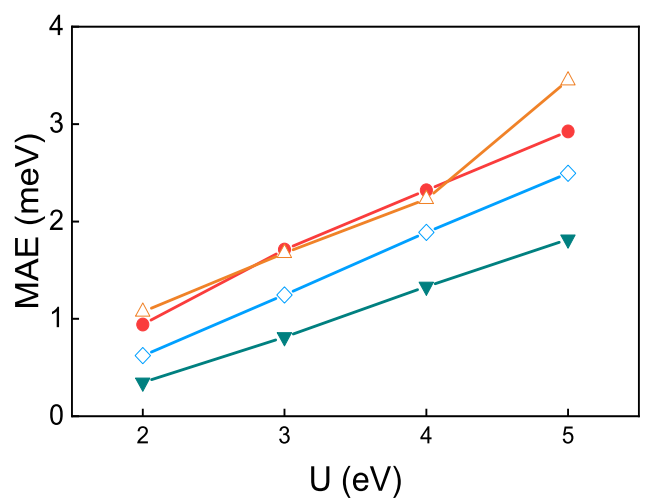

FIG. 4. The Hubbard parameter U dependence of (a) Curie temperature $T_{C}$ and (b) magnetic anisotropic energy (MAE) for $\mathrm{Cr}_{2} \mathrm{Ge}_{2} \mathrm{Te}_{6}$-relaxed, $\mathrm{Cr}_{2} \mathrm{Ge}_{2} \mathrm{Te}_{6}$-strain (3.7\%), $\mathrm{Cr}_{2} \mathrm{Ge}_{2} \mathrm{Te}_{6} / \mathrm{PtSe}_{2}$ heterostructures with Stack-1 and Stack2 , obtained by the GGA $+\mathrm{SOC}+U$ calculations.

We find that for monolayer $\mathrm{Cr}_{2} \mathrm{Ge}_{2} \mathrm{Te}_{6}, \mathrm{~J}_{x y}=\mathrm{J}_{y x}, \mathrm{~J}_{x z}$ $=\mathrm{J}_{z x}, \mathrm{~J}_{z y}=\mathrm{J}_{y z}$, and thus there is no DM interaction. This is expected, because the inversion symmetry is kept in the monolayer. For the $\mathrm{Cr}_{2} \mathrm{Ge}_{2} \mathrm{Te}_{6} / \mathrm{PtSe}_{2}$ heterostructures, the difference of the off-diagonal elements are finite values, which implies the presence of DM interaction.

The other contribution to the MAE is the SIA energy, i.e. the last term in Eq. (1). For monolayer $\mathrm{Cr}_{2} \mathrm{Ge}_{2} \mathrm{Te}_{6}$ with relaxation and strain, and $\mathrm{Cr}_{2} \mathrm{Ge}_{2} \mathrm{Te}_{6} / \mathrm{PtSe}_{2}$ heterostructure with Stack-1 and Stack-2, the SIA energy is calculated by the GGA + SOC $+U$ method, where we assume again $\mathrm{U}=4 \mathrm{eV}$. As shown in Fig. 5, the SIA energy increases from monolayer $\mathrm{Cr}_{2} \mathrm{Ge}_{2} \mathrm{Te}_{6}$ to Stack-1 and Stack-2. Comparing the SIA in Fig. 5 with the MAE in Fig. 3(b), one may note that the enhancement of the SIA and MAE is very similar.

The above DM interaction and the SIA originate from the spin-orbit coupling, which in turn contribute to the MAE. For recent magnetic semiconductor monolayers, various microscopic mechanisms of MAE are also discussed [56-58].

We also calculate the second nearest neighbor exchange coupling $\mathrm{J}_{2}$ and the nearest neighbor exchange 
TABLE III. The off-diagonal elements of exchange coupling matrix $\mathrm{J}$ (in units of meV) for monolayer $\mathrm{Cr}_{2} \mathrm{Ge}_{2} \mathrm{Te}_{6}$ with relaxation and strain, and $\mathrm{Cr}_{2} \mathrm{Ge}_{2} \mathrm{Te}_{6} / \mathrm{PtSe}_{2}$ heterostructures with Stack-1 and Stack- 2 calculated by the GGA $+\mathrm{SOC}+U$ method and the four-state method [53]. Here $\mathrm{U}=4 \mathrm{eV}$.

\begin{tabular}{|c|c|c|c|c|c|c|c|c|c|}
\hline & $\overline{J_{x y}}$ & $\mathrm{~J}_{y x}$ & $\mathrm{~J}_{x z}$ & $\mathrm{~J}_{z x}$ & $\mathrm{~J}_{y z}$ & $\overline{\mathrm{J}_{z y}}$ & $\mid \mathrm{J}_{x y}-\mathrm{J}_{y x}$ & $\mid \mathrm{J}_{x z}-\mathrm{J}_{z x}$ & $\mid \mathrm{J}_{y z}-\mathrm{J}_{z y}$ \\
\hline $\mathrm{Cr}_{2} \mathrm{Ge}_{2} \mathrm{Te}_{6}$-relax & -0.493 & -0.493 & 0.069 & 0.069 & -0.111 & -0.111 & 0.000 & 0.000 & 0.000 \\
\hline $\mathrm{Cr}_{2} \mathrm{Ge}_{2} \mathrm{Te}_{6}$-strain & -0.593 & -0.593 & -0.061 & -0.061 & 0.102 & 0.102 & 0.000 & 0.000 & 0.000 \\
\hline Stack-1 & -0.750 & -0.539 & -0.053 & 0.228 & -0.188 & -0.072 & 0.211 & 0.281 & 0.116 \\
\hline Stack-2 & 0.669 & 0.628 & -0.153 & 0.334 & 0.293 & 0.014 & 0.041 & 0.487 & 0.279 \\
\hline
\end{tabular}

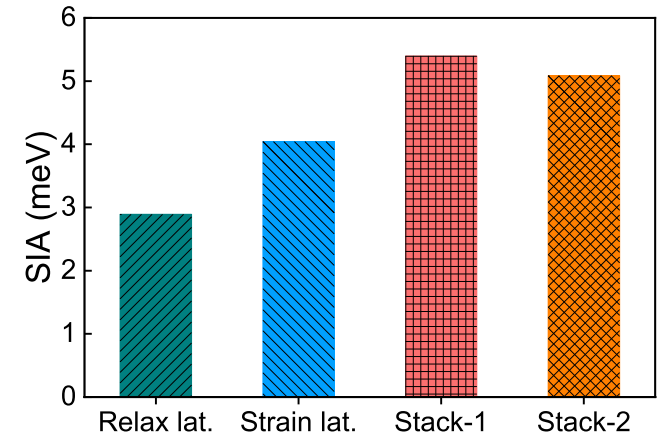

FIG. 5. The single-ion anisotropy (SIA) energy for $\quad \mathrm{Cr}_{2} \mathrm{Ge}_{2} \mathrm{Te}_{6}$-relaxed, $\quad \mathrm{Cr}_{2} \mathrm{Ge}_{2} \mathrm{Te}_{6}$-strain (3.7\%) $\mathrm{Cr}_{2} \mathrm{Ge}_{2} \mathrm{Te}_{6} / \mathrm{PtSe}_{2}$ heterostructures with Stack-1 and Stack-2 obtained by the GGA $+\mathrm{SOC}+U$ calculations, with $\mathrm{U}=4$ $\mathrm{eV}$.

coupling $\mathrm{J}_{1}$ by comparing different magnetic configurations $[54,55]$. For $2 \mathrm{D} \mathrm{Cr}_{2} \mathrm{Ge}_{2} \mathrm{Te}_{6}$ with relaxation, we obtain $\mathrm{J}_{1}=-8.38 \mathrm{meV}, \mathrm{J}_{2}=0.43 \mathrm{meV}$; for $2 \mathrm{D} \mathrm{Cr}_{2} \mathrm{Ge}_{2} \mathrm{Te}_{6}$ with strain, $\mathrm{J}_{1}=-11.64 \mathrm{meV}, \mathrm{J}_{2}=-0.06 \mathrm{meV}$; for $\mathrm{Cr}_{2} \mathrm{Ge}_{2} \mathrm{Te}_{6} / \mathrm{PtSe}$ Stack-1, J $=-13.01 \mathrm{meV}, \mathrm{J}_{2}=-0.42$ $\mathrm{meV}$; for $\mathrm{Cr}_{2} \mathrm{Ge}_{2} \mathrm{Te}_{6} / \mathrm{PtSe}_{2}$ Stack-2, $\mathrm{J}_{1}=-12.75 \mathrm{meV}$, $\mathrm{J}_{2}=-0.36 \mathrm{meV}$. Our results show that $\mathrm{J}_{2}$ was about 20 to 30 times smaller than $\mathrm{J}_{1}$ in magnitude in present cases. This is in contrast with the results in $2 \mathrm{D} \mathrm{CrI}_{3}$, where the calculated $\mathrm{J}_{2}$ is about 5 times smaller than $\mathrm{J}_{1}$ in magnitude [54]. It is noted that $T_{C}$ is determined by the leading term $\mathrm{J}_{1}$, and barely affected by small values of $\mathrm{J}_{2}$. Thus, the second nearest neighbor exchange coupling can be neglected in our case. Here we should mention that for the simplicity we applied an isotropic Heisenberg model to get $\mathrm{J}_{1}$ values by comparing the energies between different magnetic configurations. Thus, the values of $\mathrm{J}_{1}$ are approximately equal to the average of $\mathrm{J}_{x x}, \mathrm{~J}_{y y}$ and $\mathrm{J}_{z z}$.

In fact, the above results of $\mathrm{J}_{2} / \mathrm{J}_{1}$ are consistent with the magnetic anisotropy in the experiments of $2 \mathrm{D} \mathrm{CrI}_{3}$ and $\mathrm{Cr}_{2} \mathrm{Ge}_{2} \mathrm{Te}_{6}[5,6]$. For the $2 \mathrm{D} \mathrm{CrI}_{3}, \mathrm{~J}_{2} / \mathrm{J}_{1} \sim 0.2$ was obtained in the calculation [54], and thus the DM interaction can be expected, which is consistent with the large magnetic anisotropy in $2 \mathrm{D} \mathrm{CrI}_{3}$ in the experiment. In contrast, for the $2 \mathrm{D} \mathrm{Cr}_{2} \mathrm{Ge}_{2} \mathrm{Te}_{6}$, a very small $\mathrm{J}_{2} / \mathrm{J}_{1}$ $\sim 0.05$ was obtained in our calculation, which is also consistent with the small magnetic anisotropy in the experiment of $2 \mathrm{D} \mathrm{Cr}_{2} \mathrm{Ge}_{2} \mathrm{Te}_{6}$. Because the MAE in the experiment of $2 \mathrm{D} \mathrm{Cr}_{2} \mathrm{Ge}_{2} \mathrm{Te}_{6}$ is weak, we propose a scheme here to enhance MAE by constructing the heterostructure $\mathrm{Cr}_{2} \mathrm{Ge}_{2} \mathrm{Te}_{6} / \mathrm{PtSe}_{2}$, where the inversion symmetry is broken, and the DM interaction is expected.

\section{DISCUSSION}

To describe the magnetic state of $2 \mathrm{D} \mathrm{Cr}_{2} \mathrm{Ge}_{2} \mathrm{Te}_{6}$ and the heterostructures $\mathrm{Cr}_{2} \mathrm{Ge}_{2} \mathrm{Te}_{6} / \mathrm{PtSe}_{2}$, the Heisenberg model is used, as defined in Eq. (1). To estimate some magnetic properties, such as Curie temperature $T_{C}$ and exchange parameters, the approximations with classical spins are used. For example, to calculate $T_{C}$, we carry out the Monte Carlo simulations based on the Ising model. To calculate the exchange parameters $\mathrm{J}_{x x}, \mathrm{~J}_{x y}$, etc. in Eq. (1), the four-state method with four classical spin configurations are used [53].

As shown in Fig. 4(a) and (b), the Hubbard on-site Coulomb correlation $\mathrm{U}$ dependence of $T_{C}$ and MAE are calculated. For $\mathrm{U}=1 \mathrm{eV}$, our DFT results find that $2 \mathrm{D} \mathrm{Cr}_{2} \mathrm{Ge}_{2} \mathrm{Te}_{6}$ has a ferromagnetic ground state with inplane magnetization (negative MAE, not shown in Fig. 4 ), in contrast to the experimental observation that the out-of-plane magnetization was observed. Thus, this result suggests that the small $\mathrm{U}=1 \mathrm{eV}$ was not reasonable for $2 \mathrm{D} \mathrm{Cr}_{2} \mathrm{Ge}_{2} \mathrm{Te}_{6}$. Instead, our DFT calculations suggest that a large parameter $\mathrm{U}=4 \mathrm{eV}$ is reasonable for $2 \mathrm{D}$ $\mathrm{Cr}_{2} \mathrm{Ge}_{2} \mathrm{Te}_{6}$, where both $T_{C}$ and MAE are consistent with the experimental results. In Fig. 4(a) and (b), $T_{C}$ and MAE with $U$ ranging from $2-5 \mathrm{eV}$ are presented. It is shown that our conclusions, i.e., the great enhancement of Curie temperature and magnetic anisotropy in $2 \mathrm{D}$ van der Waals magnetic semiconductor heterostructures, do not change with Hubbard $\mathrm{U}$ in the range of $2-5 \mathrm{eV}$.

The great increase of $T_{C}$ in $\mathrm{Cr}_{2} \mathrm{Ge}_{2} \mathrm{Te}_{6}$ by applying tensile strain through heterostructures could be understood based on the superexchange interaction [59-61]. To deal with the superexchange interaction between the nearest neighbor $\mathrm{Cr}$ atoms we adopted the simple four-electron model [62]. In this model, four electrons $\mathrm{d}_{1}, \mathrm{p}, \mathrm{p}^{\prime}, \mathrm{d}_{2}$ are electrons of $\mathrm{Cr}_{1}$, Te (contains two $\mathrm{p}$ electrons), and $\mathrm{Cr}_{2}$ atoms, respectively. The indirect magnetic interac- 
TABLE IV. The DFT results of mixing-matrix $\left|V_{p d}\right|$ and energy difference $\left|E_{p}-E_{d}\right|$ between $5 p$ orbitals of Te and $3 d$ orbitals of $\mathrm{Cr}$ for $\mathrm{Cr}_{2} \mathrm{Ge}_{2} \mathrm{Te}_{6}$ relaxed and strained (in units of eV).

\begin{tabular}{lccccc}
\hline \hline & \multicolumn{5}{c}{$\mathrm{Cr}_{2} \mathrm{Ge}_{2} \mathrm{Te}_{6}-$ relax } \\
\cline { 2 - 6 } & $p_{y}-d_{x^{2}-y^{2}}$ & $p_{y}-d_{z^{2}}$ & $p_{x}-d_{x y}$ & $p_{y}-d_{y z}$ & $p_{z}-d_{y z}$ \\
\hline$\left|V_{p d}\right|$ & 0.7099 & 0.3583 & 0.3287 & 0.2879 & 0.2793 \\
$\left|E_{p}-E_{d}\right|$ & 0.6659 & 0.3525 & 1.1036 & 0.9681 & 1.4864 \\
\hline & & & $\mathrm{Cr}_{2} \mathrm{Ge}_{2} \mathrm{Te}_{6}$-strain & $p_{x}-d_{y z}$ \\
\cline { 2 - 6 } & $p_{x}-d_{x y}$ & $p_{y}-d_{x y}$ & $p_{y}-d_{x z}$ & 0.2561 \\
$\left|V_{p d}\right|$ & 0.4061 & 0.4021 & 0.2960 & 0.2829 & 0.2888 \\
$\left|E_{p}-E_{d}\right|$ & 0.2943 & 0.3379 & 0.4141 & 0.3030 & $d_{x y}$ \\
\hline \hline
\end{tabular}

tion between $\mathrm{Cr}_{1}$ and $\mathrm{Cr}_{2}$ can be approximately written as $J_{\text {indirect }}=\left[1 / E(\uparrow \downarrow)^{2}-1 / E(\uparrow \uparrow)^{2}\right] b^{2} J_{p d}$. The superexchange process could be divided into two intermediate processes. One intermediate process is the transfer of the p electron of Te to $\mathrm{Cr}_{1}$ site, $E(\uparrow \downarrow)$ is the energy needed if spins of $\mathrm{p}$ and $\mathrm{d}_{1}$ electrons form a singlet state, $E(\uparrow \uparrow)$ is the energy needed if spins of $\mathrm{p}$ and $\mathrm{d}_{1}$ electrons form a triplet state, and $b$ is the integral of this intermediate process. The other intermediate process is the direct exchange coupling between the remaining $\mathrm{p}^{\prime}$ electron of Te and the $\mathrm{d}_{2}$ electron of $\mathrm{Cr}_{2}$, where the antiferromagnetic p-d exchange coupling $\mathrm{J}_{p d}$ can be approximately expressed as $J_{p d}=2\left|V_{p d}\right|^{2} \frac{U}{\left(E_{p}-E_{d}\right)\left(E_{d}-E_{p}+U\right)}$ following the s-d exchange model with Schriffer-Wolff transformation [63]. For large $\mathrm{U} \gg\left|E_{p}-E_{d}\right|, \mathrm{J}_{p d}$ could be simplified to $J_{p d} \approx 2\left|V_{p d}\right|^{2} \frac{1}{\left|E_{p}-E_{d}\right|} \cdot \mathrm{V}_{p d}$ is the mixing matrix element between 5 p orbitals of Te and $3 \mathrm{~d}$ orbitals of $\mathrm{Cr}$, and $\left(E_{p}-E_{d}\right)$ is the energy difference between 5 p orbitals of $\mathrm{Te}$ and $3 \mathrm{~d}$ orbitals of $\mathrm{Cr}$. It is noted that both mixing term $\mathrm{V}_{p b}$ and energy difference $\left|E_{p}-E_{d}\right|$ are materials dependent.

By DFT calculations, we can obtain these parameters for $2 \mathrm{D} \mathrm{Cr}_{2} \mathrm{Ge}_{2} \mathrm{Te}_{6}$ relaxed and with a strain. The results of $\left|V_{p d}\right|$ and $\left|E_{p}-E_{d}\right|$ are listed in Table IV. Comparing the results of $\mathrm{Cr}_{2} \mathrm{Ge}_{2} \mathrm{Te}_{6}$ with relaxation and $\mathrm{Cr}_{2} \mathrm{Ge}_{2} \mathrm{Te}_{6}$ with strain (3.7\% tensile strain), we find that the increased antiferromagnetic coupling $\mathrm{J}_{p d}$ by strain is mainly from the decreased energy difference $\left|E_{p}-E_{d}\right|$. The decreased $\left|E_{p}-E_{d}\right|$ gives rise to the enhanced $\mathrm{J}_{p d}$, $\mathrm{J}_{\text {indirect }}$ and $T_{C}$. There is no essential difference for the $\mathrm{Cr}-\mathrm{Te}-\mathrm{Cr}$ bond angles before and after applying the strain, where $\mathrm{Cr}$-Te-Cr bond angle is 89.8 degree for $\mathrm{Cr}_{2} \mathrm{Ge}_{2} \mathrm{Te}_{6}$-relax, and 92.3 degree for $\mathrm{Cr}_{2} \mathrm{Ge}_{2} \mathrm{Te}_{6}$-strain. It is consistent with the mixing term $\left|V_{p d}\right|$ as shown in Table IV, where there is no essential difference for $\left|V_{p d}\right|$ before and after applying the strain.

As shown in Fig. 4(a), it is clear that the increased $T_{C}$ between the $\mathrm{Cr}_{2} \mathrm{Ge}_{2} \mathrm{Te}_{6}$ with relax and the $\mathrm{Cr}_{2} \mathrm{Ge}_{2} \mathrm{Te}_{6}$ with strain are nearly $U$ independent, which is consistent with the nearly $\mathrm{U}$ independent $\mathrm{J}_{p d}$. For the enhanced $T_{C}$ of heterostructures, as shown in Fig. 4 (a), an important contribution comes from the strain effect in the heterostructures. For the $T_{C}$ in heterostructures
$\mathrm{Cr}_{2} \mathrm{Ge}_{2} \mathrm{Te}_{6} / \mathrm{PtSe}$, other contribution rather than strain also appears with $\mathrm{U}>2 \mathrm{eV}$ as shown in Fig. 4(a), while the reason of such contribution needs further studies.

The MAE increases with increasing $\mathrm{U}$, as shown in Fig. 4(b). This behavior could be understood by the multiorbital electron correlations. The multiorbital Coulomb interactions between electrons with the orbitals $\mathbf{m}$ and -m and spin $\sigma$ is expressed as [16, 64]: $H_{m \sigma}=\left(U^{\prime}-\right.$ $\left.J_{H}\right) n_{m \sigma} n_{m \bar{\sigma}} \approx\left(U^{\prime}-J_{H}\right)\left(\bar{n}^{2}-\frac{1}{4} \lambda_{S O}^{2} m^{2} \sigma^{2}(\delta n)^{2}\right)$, where $\mathrm{U}$ is the on-site Coulomb interactions within the same orbitals, $\mathrm{U}^{\prime}$ is the on-site Coulomb interactions between different orbitals, and $\mathrm{J}_{H}$ demotes the Hund coupling. In the atomic limit, these parameters satisfy the relation $\mathrm{U}=\mathrm{U}^{\prime}+2 \mathrm{~J}_{H}[65] . \quad \lambda_{S O}$ is the SOC parameter, giving rise to the band splitting due to spin-orbit coupling. To compensate the increased energy due to the multiorbital Coulomb interactions, the SOC will increase with increasing U. Such enhancement of SOC due to the electron correlations has been discussed in the large topological band gap [16] and the enhancement of spin Hall effect $[66,67]$. Because the MAE is originated from the SOC, MAE will also increase with increasing $U$.

When growing two materials together, two kinds of junctions can be considered. One is the incoherent junction, where each layer of the junction keeps their original lattice constants [37], and there is little strain at the junction. Another is the coherent junction, where the lattice constant of each layer is forced to be the same, and the strain is expected at the junction. In this work, the coherent junction is considered, and the strain at the junction is found to play an important role in enhancing the magnetic anisotropy and Curie temperature of heterostructures. Our results suggest that it is better to apply the coherent junctions to manipulate the magnetic properties of heterostructures.

\section{SUMMARY}

In this paper, we have proposed that the magnetic anisotropic energy (MAE) of 2D ferromagnetic semiconductor can be strongly enhanced in heterostructures with a nonmagnetic semiconductor monolayer with large spin-orbit coupling. Based on the density functional 
theory calculations, we have demonstrated this idea in the bilayer heterostructure $\mathrm{Cr}_{2} \mathrm{Ge}_{2} \mathrm{Te}_{6} / \mathrm{PtSe}_{2}$. The results show that the MAE of $\mathrm{Cr}_{2} \mathrm{Ge}_{2} \mathrm{Te}_{6}$ is enhanced by $70 \%$, and its Curie temperature is increased to larger than $600 \mathrm{~K}$ far beyond room temperature. By means of a model Hamiltonian analysis, it is shown that both the Dzyaloshinskii-Moriya interaction and the single-ion anisotropy contribute to the enhancement of the MAE. Based on the superexchange picture, the great enhancement of $T_{C}$ is partially attributed to the decreased energy difference between $3 \mathrm{~d}$ orbitals of $\mathrm{Cr}$ and $5 \mathrm{p}$ orbitals of Te. Our present study gives a deeper understanding on the mechanism of enhancement of magnetism in 2D van der Waals magnetic/nonmagnetic heterostructures and will spur further experimental studies of the $2 \mathrm{D}$ magnetic semiconductor systems.

\section{ACKNOWLEDGMENTS}

The authors acknowledge Q. B. Yan, Z. G. Zhu, and Z. C. Wang for many valuable discussions. This paper is supported in part by the National Key R\&D Program of China (Grant No. 2018YFA0305800), the Strategic Priority Research Program of the Chinese Academy of Sciences (Grant No. XDB28000000), the National Natural Science Foundation of China (Grant No.11834014), and Beijing Municipal Science and Technology Commission (Grant No. Z190011). B.G. is also supported by the National Natural Science Foundation of China (Grant No.Y81Z01A1A9), the Chinese Academy of Sciences (Grant No. Y929013EA2), the University of Chinese Academy of Sciences (Grant No. 110200M208), the Strategic Priority Research Program of Chinese Academy of Sciences (Grant No.XDB33000000),and the Beijing Natural Science Foundation (Grant No. Z190011).
[1] D. Kennedy and C. Norman, What don't we know?, Science 309, 75 (2005).

[2] K. S. Burch, D. Mandrus, and J.-G. Park, Magnetism in two-dimensional van der waals materials, Nature 563, 47 (2018).

[3] J. L. Miller, Ferromagnetism found in two-dimensional materials, Phys. Today 70, 16 (2017).

[4] N. Sethulakshmi, A. Mishra, P. Ajayan, Y. Kawazoe, A. K. Roy, A. K. Singh, and C. S. Tiwary, Magnetism in two-dimensional materials beyond graphene, Mater. Today 27, 107 (2019).

[5] C. Gong, L. Li, Z. Li, H. Ji, A. Stern, Y. Xia, T. Cao, W. Bao, C. Wang, Y. Wang, Z. Q. Qiu, R. J. Cava, S. G. Louie, J. Xia, and X. Zhang, Discovery of intrinsic ferromagnetism in two-dimensional van der Waals crystals, Nature 546, 265 (2017).

[6] B. Huang, G. Clark, E. Navarro-Moratalla, D. R. Klein, R. Cheng, K. L. Seyler, D. Zhong, E. Schmidgall, M. A. McGuire, D. H. Cobden, W. Yao, D. Xiao, P. JarilloHerrero, and $\mathrm{X}$. Xu, Layer-dependent ferromagnetism in a van der Waals crystal down to the monolayer limit, Nature 546, 270 (2017).

[7] Y. Deng, Y. Yu, Y. Song, J. Zhang, N. Z. Wang, Z. Sun, Y. Yi, Y. Z. Wu, S. Wu, J. Zhu, J. Wang, X. H. Chen, and Y. Zhang, Gate-tunable room-temperature ferromagnetism in two-dimensional $\mathrm{Fe}_{3} \mathrm{GeTe}_{2}$, Nature 563, 94 (2018).

[8] M. Bonilla, S. Kolekar, Y. Ma, H. C. Diaz, V. Kalappattil, R. Das, T. Eggers, H. R. Gutierrez, M.-H. Phan, and M. Batzill, Strong room-temperature ferromagnetism in $\mathrm{VSe}_{2}$ monolayers on van der waals substrates, Nat. Nanotechnol. 13, 289 (2018).

[9] D. J. O'Hara, T. Zhu, A. H. Trout, A. S. Ahmed, Y. K. Luo, C. H. Lee, M. R. Brenner, S. Rajan, J. A. Gupta, D. W. McComb, and R. K. Kawakami, Room temperature intrinsic ferromagnetism in epitaxial manganese selenide films in the monolayer limit, Nano Lett. 18, 3125 (2018).

[10] S. Zheng, C. Huang, T. Yu, M. Xu, S. Zhang, H. Xu, Y. Liu, E. Kan, Y. Wang, and G. Yang,
High-temperature ferromagnetism in an $\mathrm{Fe}_{3} \mathrm{P}$ monolayer with a large magnetic anisotropy, J. Phys. Chem. Lett. 10, 2733 (2019).

[11] J.-U. Lee, S. Lee, J. H. Ryoo, S. Kang, T. Y. Kim, P. Kim, C.-H. Park, J.-G. Park, and H. Cheong, Ising-type magnetic ordering in atomically thin $\mathrm{FePS}_{3}$, Nano Lett. 16, 7433 (2016).

[12] N. D. Mermin and H. Wagner, Absence of ferromagnetism or antiferromagnetism in one- or two-dimensional isotropic Heisenberg models, Phys. Rev. Lett. 17, 1133 (1966).

[13] C. Xu, J. Feng, H. Xiang, and L. Bellaiche, Interplay between Kitaev interaction and single ion anisotropy in ferromagnetic $\mathrm{CrI}_{3}$ and $\mathrm{CrGeTe}_{3}$ monolayers, npj Comput. Mater. 4, 57 (2018).

[14] X. Li, B. Dong, X. Sun, H. Wang, T. Yang, G. Yu, and Z. V. Han, Perspectives on exfoliated two-dimensional spintronics, J. Semicond. 40, 081508 (2019).

[15] S. Jiang, J. Shan, and K. F. Mak, Electric-field switching of two-dimensional van der Waals magnets, Nat. Mater. 17, 406 (2018).

[16] J.-Y. You, Z. Zhang, B. Gu, and G. Su, Twodimensional room-temperature ferromagnetic semiconductors with quantum anomalous hall effect, Phys. Rev. Appl 12, 024063 (2019).

[17] X.-J. Dong, J.-Y. You, B. Gu, and G. Su, Strain-induced room-temperature ferromagnetic semiconductors with large anomalous hall conductivity in two-dimensional $\mathrm{Cr}_{2} \mathrm{Ge}_{2} \mathrm{Se}_{6}$, Phys. Rev. Appl 12, 014020 (2019).

[18] L. Webster and J.-A. Yan, Strain-tunable magnetic anisotropy in monolayer $\mathrm{CrCl}_{3}, \mathrm{CrBr}_{3}$, and $\mathrm{CrI}_{3}$, Phys. Rev. B 98, 144411 (2018).

[19] Y. Liu, Y. Huang, and X. Duan, Van der Waals integration before and beyond two-dimensional materials, Nature 567, 323 (2019).

[20] M. Gibertini, M. Koperski, A. F. Morpurgo, and K. S. Novoselov, Magnetic 2D materials and heterostructures, Nat. Nanotechnol. 14, 408 (2019).

[21] C. Jin, E. Y. Ma, O. Karni, E. C. Regan, F. Wang, and T. F. Heinz, Ultrafast dynamics in van der Waals het- 
erostructures, Nat. Nanotechnol. 13, 994 (2018).

[22] K. S. Novoselov, A. Mishchenko, A. Carvalho, and A. H. C. Neto, 2D materials and van der Waals heterostructures, Science 353, 9439 (2016).

[23] X. Liu and M. C. Hersam, Interface characterization and control of 2D materials and heterostructures, Adv. Mater. 30, 1801586 (2018).

[24] E. Pomerantseva and Y. Gogotsi, Twodimensional heterostructures for energy storage, Nature Energy 2, 17089 (2017).

[25] M.-Y. Li, C.-H. Chen, Y. Shi, and L.-J. Li, Heterostructures based on two-dimensional layered materials and their potential applications, Mater. Today 19, 322 (2016).

[26] P. Solís-Fernández, M. Bissett, and H. Ago, Synthesis, structure and applications of graphene-based 2D heterostructures, Chem. Soc. Rev. 46, 4572 (2017).

[27] J. Kang, J. Li, S.-S. Li, J.-B. Xia, and L.-W. Wang, Electronic structural moiré pattern effects on $\mathrm{MoS}_{2} / \mathrm{MoSe}_{2}$ 2D heterostructures, Nano Lett. 13, 5485 (2013).

[28] B. Cho, J. Yoon, S. K. Lim, A. R. Kim, D.-H. Kim, S.-G. Park, J.-D. Kwon, Y.-J. Lee, K.-H. Lee, B. H. Lee, H. C. Ko, and M. G. Hahm, Chemical sensing of $2 \mathrm{D}$ graphene/ $\mathrm{MoS}_{2}$ heterostructure device, ACS Appl. Mater. Interfaces 7, 16775 (2015).

[29] J. Zhang, L. Du, S. Feng, R.-W. Zhang, B. Cao, C. Zou, Y. Chen, M. Liao, B. Zhang, S. A. Yang, G. Zhang, and T. Yu, Enhancing and controlling valley magnetic response in $\mathrm{MoS}_{2} / \mathrm{WS}_{2}$ heterostructures by all-optical route, Nat. Commun. 10, 4226 (2019).

[30] C. Gong, E. M. Kim, Y. Wang, G. Lee, and X. Zhang, Multiferroicity in atomic van der Waals heterostructures, Nat. Commun. 10, 2657 (2019).

[31] Y. Zhu, X. Wang, and W. Mi, Tunable electronic structure and magnetic anisotropy of two dimensional van der Waals $\mathrm{GeS} / \mathrm{FeCl}_{2}$ multiferroic heterostructures, J. Mater. Chem. C 7, 2049 (2019).

[32] D. Zhong, K. L. Seyler, X. Linpeng, R. Cheng, N. Sivadas, B. Huang, E. Schmidgall, T. Taniguchi, K. Watanabe, M. A. McGuire, W. Yao, D. Xiao, K.M. C. Fu, and X. Xu, Van der Waals engineering of ferromagnetic semiconductor heterostructures for spin and valleytronics, Sci. Adv. 3, e1603113 (2017).

[33] X.-R. Chen, W. Chen, L. B. Shao, and D. Y. Xing, Engineering chiral edge states in two-dimensional topological insulator/ferromagnetic insulator heterostructures, Phys. Rev. B 99, 085417 (2019).

[34] W. Sun, W. Wang, D. Chen, Z. Cheng, and Y. Wang, Valence mediated tunable magnetism and electronic properties by ferroelectric polarization switching in $2 \mathrm{D} \mathrm{FeI}_{2} / \mathrm{In}_{2} \mathrm{Se}_{3}$ van der Waals heterostructures, Nanoscale 11, 9931 (2019).

[35] K. Takiguchi, T. Chiba, T. Koyama, D. Chiba, M. Tanaka, et al., Giant gate-controlled proximity magnetoresistance in semiconductor-based ferromagneticnon-magnetic bilayers, Nat. Phys. 15, 1134 (2019).

[36] L. Zhang, Z. Zhou, Y. Zhang, B. Peng, W. Ren, Z.-G. Ye, and M. Liu, Tuning the magnetic anisotropy of $\mathrm{Fe}_{3} \mathrm{O}_{4} / \mathrm{Pt}$ heterostructures fabricated by atomic layer deposition with in situ magnetic field, IEEE Transactions on Magnetics 55, 1 (2019).

[37] A. Koma, Van der Waals epitaxy a new epitaxial growth method for a highly lattice-mismatched system, Thin Solid Films 216, 72 (1992).
[38] X. Li, M.-W. Lin, J. Lin, B. Huang, A. A. Puretzky, C. Ma, K. Wang, W. Zhou, S. T. Pantelides, M. Chi, I. Kravchenko, J. Fowlkes, C. M. Rouleau, D. B. Geohegan, and K. Xiao, Two-dimensional GaSe/MoSe 2 misfit bilayer heterojunctions by van der Waals epitaxy, Sci. Adv. 2, e1501882 (2016).

[39] A. Ghasemi, D. Kepaptsoglou, P. L. Galindo, Q. M. Ramasse, T. Hesjedal, and V. K. Lazarov, Van der Waals epitaxy between the highly lattice mismatched $\mathrm{Cu}$-doped FeSe and $\mathrm{Bi}_{2} \mathrm{Te}_{3}$, NPG Asia Mater. 9, e402 (2017).

[40] A. Ciarrocchi, A. Avsar, D. Ovchinnikov, and A. Kis, Thickness-modulated metal-to-semiconductor transformation in a transition metal dichalcogenide, Nat. Commun. 9, 919 (2018).

[41] W. Chen, Z. Sun, Z. Wang, L. Gu, X. Xu, $\mathrm{S}$. $\mathrm{Wu}$, and $\mathrm{C}$. Gao, Direct observation of van der Waals stacking-dependent interlayer magnetism, Science 366, 983 (2019).

[42] J. Li, X. Yang, Y. Liu, B. Huang, R. Wu, Z. Zhang, B. Zhao, H. Ma, W. Dang, Z. Wei, K. Wang, Z. Lin, X. Yan, M. Sun, B. Li, X. Pan, J. Luo, G. Zhang, Y. Liu, Y. Huang, X. Duan, and X. Duan, General synthesis of two-dimensional van der Waals heterostructure arrays, Nature 579, 368 (2020).

[43] G. Kresse and J. Furthmller, Efficient iterative schemes for ab initio total-energy calculations using a plane-wave basis set, Phys. Rev. B 54, 11169 (1996).

[44] S. Grimme, Semiempirical GGA-type density functional constructed with a long-range dispersion correction, J. Comput. Chem. 27, 1787 (2006).

[45] J. Liu, Q. Sun, Y. Kawazoe, and P. Jena, Exfoliating biocompatible ferromagnetic $\mathrm{Cr}$-trihalide monolayers, Phys. Chem. Chem. Phys. 18, 8777 (2016).

[46] V. Carteaux, D. Brunet, G. Ouvrard, and G. Andre, Crystallographic, magnetic and electronic structures of a new layered ferromagnetic compound $\mathrm{Cr}_{2} \mathrm{Ge}_{2} \mathrm{Te}_{6}$, J. Phys.: Condens. Matter 7, 69 (1995).

[47] J. Zeisner, A. Alfonsov, S. Selter, S. Aswartham, M. P. Ghimire, M. Richter, J. van den Brink, B. Buchner, and V. Kataev, Magnetic anisotropy and spin-polarized twodimensional electron gas in the van der Waals ferromagnet $\mathrm{Cr}_{2} \mathrm{Ge}_{2} \mathrm{Te}_{6}$, Phys. Rev. B 99, 165109 (2019).

[48] Y. Liu and C. Petrovic, Critical behavior of quasi-twodimensional semiconducting ferromagnet $\mathrm{Cr}_{2} \mathrm{Ge}_{2} \mathrm{Te}_{6}$, Phys. Rev. B 96, 054406 (2017).

[49] N. Janša, A. Zorko, M. Gomilšek, M. Pregelj, K. W. Krmer, D. Biner, A. Biffin, C. Regg, and M. Klanjšek, Observation of two types of fractional excitation in the Kitaev honeycomb magnet, Nat. Phys. 14, 786 (2018).

[50] A. Fernández-Pacheco, E. Vedmedenko, F. Ummelen, R. Mansell, D. Petit, and R. P. Cowburn, Symmetrybreaking interlayer Dzyaloshinskii-Moriya interactions in synthetic antiferromagnets, Nat. Mater. 18, 679 (2019).

[51] A. Hrabec, N. A. Porter, A. Wells, M. J. Benitez, G. Burnell, S. McVitie, D. McGrouther, T. A. Moore, and C. H. Marrows, Measuring and tailoring the DzyaloshinskiiMoriya interaction in perpendicularly magnetized thin films, Phys. Rev. B 90, 020402 (2014).

[52] S. Shi, S. Liang, Z. Zhu, K. Cai, S. D. Pollard, Y. Wang, J. Wang, Q. Wang, P. He, J. Yu, G. Eda, G. Liang, and H. Yang, All-electric magnetization switching and dzyaloshinskii-moriya interaction in $\mathrm{WTe}_{2}$ /ferromagnet heterostructures, Nat. Nanotechnol. 14, 945 (2019). 
[53] H. Xiang, C. Lee, H.-J. Koo, X. Gong, and M.H. Whangbo, Magnetic properties and energy-mapping analysis, Dalton Trans. 42, 823 (2013).

[54] V. K. Gudelli and G.-Y. Guo, Magnetism and magnetooptical effects in bulk and few-layer $\mathrm{CrI}_{3}$ : a theoretical GGA + U study, New J. Phys. 21, 053012 (2019).

[55] N. Sivadas, S. Okamoto, X. Xu, C. J. Fennie, and D. Xiao, Stacking-dependent magnetism in bilayer $\mathrm{CrI}_{3}$, Nano Lett. 18, 7658 (2018).

[56] T. Olsen, Theory and simulations of critical temperatures in $\mathrm{CrI}_{3}$ and other $2 \mathrm{D}$ materials: easy-axis magnetic order and easy-plane Kosterlitz-Thouless transitions, MRS Communications 9, 1142 (2019).

[57] S. Biswas, Y. Li, S. M. Winter, J. Knolle, and R. Valentí, Electronic properties of $\mathrm{RuCl}_{3}$ in proximity to graphene, Phys. Rev. Lett. 123, 237201 (2019).

[58] Y. Liu and C. Petrovic, Anisotropic magnetic entropy change in $\mathrm{Cr}_{2} \mathrm{X}_{2} \mathrm{Te}_{6}(\mathrm{X}=\mathrm{Si}$ and $\mathrm{Ge})$, Phys. Rev. Materials 3, 014001 (2019).

[59] J. B. Goodenough, Theory of the role of covalence in the perovskite-type manganites[La, $\mathrm{M}(\mathrm{II})] \mathrm{MnO}_{3}$, Phys. Rev. 100, 564 (1955).
[60] J. Kanamori, Crystal distortion in magnetic compounds, J. Appl. Phys. 31, S14 (1960).

[61] P. W. Anderson, New approach to the theory of superexchange interactions, Phys. Rev. 115, 2 (1959).

[62] K.-M. Qian. D.-Sh. Dai, Ferromagnetism: Volume I, (Science Press, 2017) pp. 193-198.

[63] J. R. Schrieffer and P. A. Wolff, Relation between the Anderson and Kondo Hamiltonians, Phys. Rev. 149, 491 (1966).

[64] M. C. Nguyen, Y. Yao, C.-Z. Wang, K.-M. Ho, and V. P. Antropov, Magnetocrystalline anisotropy in cobalt based magnets: a choice of correlation parameters and the relativistic effects, J. Phys.: Condens. Matter 30, 195801 (2018).

[65] S. Maekawa, Introduction, in Physics of Transition Metal Oxides (Springer Berlin Heidelberg, Berlin, Heidelberg, 2004) pp. 1-35.

[66] B. Gu, J.-Y. Gan, N. Bulut, T. Ziman, G.-Y. Guo, N. Nagaosa, and S. Maekawa, Quantum renormalization of the Spin Hall effect, Phys. Rev. Lett. 105, 086401 (2010).

[67] G.-Y. Guo, S. Maekawa, and N. Nagaosa, Enhanced Spin Hall effect by resonant skew scattering in the orbital-dependent Kondo effect, Phys. Rev. Lett. 102, 036401 (2009). 